\title{
El significado filosófico de la teoría cuántica de campos
}

\section{The Philosophical Meaning of Quantum Field Theory}

\author{
RAFAEL ANDRÉS ALEMAÑ BERENGUER \\ Universidad Miguel Hernández de Elche
}

Recibido: 22-02-2010 Aprobado definitivamente: 14-06-2010

RESUMEN

Los abundantes análisis filosóficos sobre la física cuántica no se han visto en general acompañados por una consideración equiparable hacia su prolongación natural en la teoría cuántica de campos. Esta teoría se ha revelado en su versión electromagnética como una de las herramientas predictivas más precisas de la ciencia física. No obstante, sus cimientos conceptuales siguen siendo altamente controvertidos y cabe dudar si una ampliación de su formalismo conducirá a la tan deseada unificación de las fuerzas fundamentales, y por ende, a una comprensión global de las propiedades básicas de la naturaleza.

\section{PALABRAS CLAVE \\ FILOSOFÍA DE LA FÍSICA, TEORÍA CUÁNTICA DE CAMPOS, UNIFICACIÓN DE FUERZAS, PREDICCIÓN}

\begin{abstract}
The abundant philosophical analyses on quantum physics have not been generally followed by an equivalent consideration toward their natural continuation in the quantum field theory. This theory is regarded in its electromagnetic version as one of the best predictive tools in physical science. Nevertheless, its conceptual foundations are still surrounded by controversy and it is doubtful if an extension of its formalism will lead us to the unification of the four basic forces, and therefore to a global understanding of the basic properties of nature.
\end{abstract}

KEYWORDS

PHILOSOPHY OF PHYSICS, QUANTUM FIELD THEORY, UNIFICATION OF FORCES, PREDICTION

(C) Contrastes. Revista Internacional de Filosofía, vol. XVI (2011), pp. 27-47. ISSN: 1136-4076

Departamento de Filosofía, Universidad de Málaga, Facultad de Filosofía y Letras Campus de Teatinos, E-29071 Málaga (España) 


\section{INTRODUCCIÓN}

DE ENTRE LAS TEORÍAS FísICAS juzgadas revolucionarias en la ciencia del siglo XX, sin duda la más impactante por su significación y sus implicaciones fue la teoría cuántica. La teoría cuántica del campo electromagnético, la electrodinámica cuántica, resulta ser la más exitosa teoría física de la historia, si por éxito entendemos la coincidencia entre las predicciones calculadas y las mediciones experimentales. Sus pronósticos se cumplen con gran exactitud en muy diversos terrenos experimentales: espectros atómicos, colisiones y dispersiones, emisiones de microondas, radiaciones de frenado, aniquilaciones entre partículas, láseres, metrología, detección de radiofrecuencias, métodos numéricos, la física de aceleradores y muchos otros (Karshenboim 2005, Karshenboim et al 2005, Lee et al 2005, Pachucki 2005, Sick 2005, Cladé et al 2006, Gabrielse et al 2006, Odom et al 2006, Hagiwara et al 2007).

Todo invitaba a pensar que por este camino lograría avanzarse con paso firme hacia la posterior conjunción entre la gravitación relativista-la relatividad general- y las otras tres fuerzas fundamentales (electromagnetismo, fuerza nuclear fuerte y fuerza nuclear débil), una vez fuesen embridadas en el marco de los citados campos cuánticos. ${ }^{1}$

La realidad, empero, se ha mostrado esquiva con tales esperanzas. Más de sesenta años después del desarrollo de la teoría cuántica de campos, ni se ha conseguido la tan anhelada unificación ni estamos siquiera seguros de que la física cuántica resulte compatible en sentido estricto con la relatividad especial (no digamos ya la general). La inmensa mayoría de los textos sobre teoría cuántica de campos están destinados a la formación de especialistas, de modo que eluden los debates sobre sus fundamentos. Y los escasos trabajos semidivulgativos al respecto raramente abandonan una línea laudatoria hacia los logros predictivos de la teoría -que, en algunos casos, se muestran ciertamente admirables- sin apenas atisbo de juicio crítico. ${ }^{2}$

El presente trabajo pretende constituirse como una minúscula aportación en este terreno, enjuiciando la teoría cuántica de campos no solo por sus éxitos -nada desdeñables, ya se ha dicho- sino también por la solidez de sus cimientos, la coherencia de sus interpretaciones y las perspectivas que promete hacia el futuro. Pues la fertilidad potencial de una teoría, más allá de sus triunfos pretéritos, depende también de la claridad de sus fundamentos y de la consistencia lógica de sus métodos.

1 La electrodinámica cuántica ha alcanzado precisiones que rondan una parte en $10^{11}$, equiparables a los mejores resultados de la relatividad general. La relatividad especial, comparativamente más sencilla, exhibe precisiones de entorno a una parte en $10^{21}$; véase Haugan y Will (1989).

2 Excepto en contados - pero muy notables- casos, como en Penrose (2006). 


\section{EL ARMAZÓN DE LOS CAMPOS CUÁNTICOS}

Como punto de partida, las teorías cuánticas de campos al uso toman el espacio-tiempo de Minkowski, donde el grupo de simetría es el grupo de Poincaré (traslaciones y rotaciones de Lorentz). Los campos cuánticos no son operadores ordinarios, sino distribuciones -en el sentido matemático de Schwarz- cuyos valores son operadores. El hecho de manejar distribuciones matemáticas y no funciones, implica que los operadores cuánticos de campo no resultan definibles en un punto concreto, sino en un entorno espacio-temporal de extensión finita. Naturalmente, los productos de estos operadores en un punto -a diferencia de la teoría clásica de campos- tampoco están definidos, y no es de extrañar que aparezcan divergencias infinitas al aplicar este formalismo tan espinosamente construido.

El deseo de incorporar la causalidad espacio-temporal junto con las simetrías del grupo de Poincaré, aconseja escoger una formulación lagrangiana para las teorías cuánticas de campos. Para ello se mantiene la forma de la densidad lagrangiana de un campo clásico (que en general es una combinación local de los campos presentes y sus derivadas espacio-temporales) y se sustituyen los campos clásicos $\varphi(\xi)$ por los operadores cuánticos $\phi(\xi)$. Cuando aplicamos todo este aparato formal a la interacción entre los campos de materia eléctricamente cargados (digamos, electrones) y los campos electromagnéticos (generalmente a través de un potencial-vector) llegamos a la electrodinámica cuántica, que es una teoría gauge local y abeliana. Estas tres últimas palabras encierran un riquísimo significado físico, a saber: (1) Todas las cantidades observables quedan invariantes bajo un cambio de gauge. De ello se colige que son las clases de equivalencia de los campos vectoriales (campos que sólo difieren entre sí por una transformación gauge) las que poseen relevancia física, no los campos individuales. (2) Su carácter local implica que las transformaciones gauge pueden variar de un lugar a otro, es decir, son dependientes del punto espacio-temporal escogido. Las interacciones son locales -no hay acción a distancia- y las señales físicas se propagan a velocidad finita. (3) Si aplicamos dos transformaciones gauge sucesivas el resultado es independiente del orden de su aplicación. En eso consiste la propiedad abeliana.

Pocas veces se llama la atención sobre el hecho de que la descripción relativista de las partículas cuánticas fue obra de Wigner, y no guarda relación directa con la idea de los campos cuánticos (Wigner, 1939). En general, no hay una relación directa entre el número de campos presentes y el número de partículas descritas por una teoría cuántica de campos. La correspondencia entre campos y partículas sólo es unívoca ${ }^{3}$-cada campo va ligado con un tipo

3 Excepto los campos "fantasmas" (ghosts). 
de partícula- en el caso libre de interacciones, o cuando las ecuaciones de la teoría se expresan desde el inicio mediante una serie de potencias.

Se admite también que un campo cuántico posee un conjunto característico de números cuánticos que desempeñan el papel de "cargas" en las interacciones presentes (Jost 1965, Streater y Wightman 1980). Si la lagrangiana de un campo cuántico obedece una simetría interna (como el isospín) el número cuántico relacionado con ella obedece una ley de conservación impuesta sobre las reacciones entre partículas asociadas con dicho campo. ${ }^{4}$

La eliminación de las cantidades infinitas, o "divergencias", en las teorías cuánticas de campo se consigue al precio de introducir grados de libertad (parámetros con valores sin fijar) de dudosa interpretación física. Por desgracia estos grados de libertad no físicos parecen ser imprescindibles para preservar la simetría gauge local y la simetría espacio-temporal del grupo de Poincaré. La apelación a esos grados adicionales de libertad, nos aboca a un espacio ampliado de estados con una métrica indefinida. El espacio de estados genuinamente físicos, por su parte, configura un subespacio con métrica definida positiva, lo que evita la aparición de probabilidades negativas (es decir, la matriz de densidad $S$ permanece unitaria). Considerando el asunto más de cerca, se observa que en la electrodinámica cuántica -una teoría abeliana- los grados de libertad no físicos provienen en exclusiva del gauge del vector potencial del campo electromagnético. Por el contrario, en las teorías no abelianas -como la de simetría $S U(2)$ - surgen por añadidura campos cuánticos escalares sometidos a una estadística fermiónica, ${ }^{5}$ rompiendo con ello la conexión espín-estadística que rige en el caso de los grados de libertad verdaderamente físicos.

\section{LAS TÉCNICAS DE RENORMALIZACIÓN.}

A juicio de numerosos investigadores, siendo muy importante no basta sólo con eliminar los infinitos; había que lograrlo mediante métodos matemáticos legítimos, pues de lo contrario toda la teoría amenazaba con quedar sospechosamente viciada. Dado que desconocemos la masa y la carga "desnudas" del electrón (esto es, sin la adición de la masa electromagnética ni el apantallamiento de las partículas virtuales), nada nos impide asignarle un valor arbitrario de modo que la suma final coincida con los datos experimentales. Asignando una masa y

4 También tenemos otras funciones de correlaciones llamadas funciones de Schwinger. Para campos bosónicos se definen sobre un espacio abstracto de distribuciones. Con fermiones se obtienen de la integración de Berezin (1966), definida sobre un álgebra de Grassmann.

5 Son los campos "fantasmas" y "antifantasmas" de Faddeev-Popov (Faddeev \& Slavnov, 1991). 
una carga infinitas al electrón, y escogiendo bien el orden de infinitud para que cancele el de la serie de términos perturbativos, podemos obtener un resultado finito arbitrario. Las operaciones con cantidades infinitas se suelen considerar matemáticamente ilícitas dado que generan indeterminaciones, aunque eso mismo nos brinda la posibilidad de asignar a esa magnitud indeterminada el valor fenomenológico de la masa y la carga del electrón. Así se hizo con gran éxito práctico ${ }^{6}$ tan grande de hecho que acalló momentáneamente a la mayoría de quienes dudaban de la solidez matemática del método, y temían que tales reservas acabaran comprometiendo la fiabilidad misma de la electrodinámica cuántica (Lewis 1948, Epstein 1948).

El hecho palmario de que las predicciones coincidiesen extremadamente bien con los experimentos, no impresionaba demasiado a Dirac, quien se refería a las reglas de renormalización diciendo Dirac (1978, p. 20): «[...] incluso aunque puedan conducir a resultados concordantes con las observaciones, son reglas artificiales, yo simplemente no puedo aceptar que los fundamentos actuales [de la teoría cuántica relativista] sean correctos». Y en su último artículo no dejó de repetir ${ }^{7}$ (Dirac 1987, p. 196): «Quiero enfatizar que muchas de estas modernas teorías cuánticas de campos no son fiables en absoluto, incluso aunque mucha gente esté trabajando en ellas y su labor obtenga a veces resultados detallados».

Resulta difícil, por ejemplo, vincular los operadores de la electrodinámica cuántica con las habituales funciones de puntos del espacio-tiempo minkowskiano, aunque de hecho las reglas de renormalización los tratan como si así fuesen (Bogolubov et al 1975, sec. 10.4; Itzykson y Zuber 1980, sec. 6.1). Esas mismas reglas se emplean para restar divergencias manipulando integrales mal definidas con la coartada de la teoría de distribuciones y de funciones generalizadas (Schwartz 1945, Gel'fand y Shilov 1964, Gel'fand y Vilenkin 1968). Sin embargo, pese a la frecuente apelación a la legitimidad matemática que supuestamente proporcionan, tanto las distribuciones como las funciones generalizadas son funcionales lineales continuos, que por su propia naturaleza excluyen el tratamiento de expresiones no lineales. Dado que los términos no lineales caracterizan precisamente los campos en interacción -que son los fenómenos físicamente interesantes- ahí se halla la dificultad esencial de toda teoría cuántica que aspire a describir la naturaleza de modo fructífero.

Pero concedamos por un momento que la manipulación de las magnitudes divergentes suministra resultados finitos, los cuales se supone que constituyen los términos de un desarrollo perturbativo de los elementos de la matriz de

6 Para un interesante relato histórico sobre la acogida de los métodos de renormalización en la comunidad científica, es muy aconsejable consultar Pais (1986), pp. 360-392.

7 Véase Krisch (1987), p. 50. 
dispersión (matriz $S$ ) para diversos procesos. Entonces echamos en falta una prueba de que dichos elementos de la matriz $S$ existan de veras en un sentido matemático bien definido. Pese a ello, si conservamos nuestro ánimo intacto, renunciamos a la convergencia de los desarrollos perturbativos y aceptamos en cambio la conjetura usual en electrodinámica cuántica (Dyson 1952) -y también en otras teorías cuánticas de campos- de que nos enfrentamos con "series asintóticas".

Ahora bien, privados de una caracterización sólida de aquellas funciones con respecto a las cuales los desarrollos perturbativos se suponen asintóticos (es decir, los elementos de la matriz $S$ ) el concepto mismo de serie asintótica resulta controvertido. El criterio de sumabilidad de Borel puede emplearse para reconstruir una función partiendo de una serie divergente de potencias (Hardy 1949, Sokal 1980), pero los requisitos para su aplicabilidad no se satisfacen en la electrodinámica cuántica.

Las técnicas de Feynman destinadas a librar la electrodinámica cuántica de los infinitos (Feynman 1948, 1949, 1962) no eran menos arbitrarias que las de Schwinger y Tomonaga. La idea del estadounidense consistía en realizar un truncamiento de la serie de términos perturbativos justo antes de que su valor se desbocase hacia el finito. Pero careciendo de un criterio seguro mediante el cual discernir en qué termino de la serie detenerse, este procedimiento no dejaba de ser igualmente artificioso. Peor aún, de sus intentos de sustituir las deltas de Dirac por otro tipo de funciones menos problemáticas, quedó claro que las divergencias de la electrodinámica cuántica provenían tanto de la ausencia de una cota superior para la energía de los cuantos virtuales, como del carácter local de la interacción entre los campos. ${ }^{8}$

¿Qué pensar entonces de los métodos de renormalización? Su existencia ha permitido a los experimentalistas alcanzar grados de precisión desconocidos en la permanente contienda entre teorías y contrastaciones. No obstante, la ausencia de unos fundamentos matemáticos sólidos mantenía viva la inquietud incluso en físicos geniales, como el propio Dirac, muy comprometidos con el mundo cuántico: ${ }^{9}$

Hubo un tiempo en el que todas las teorías físicas se basaban en matemáticas intrínsecamente correctas. No pretendo decir con esto que los físicos siempre

8 Como se expone nítidamente en Bogoliubov y Shirkov (1959), p. 299.

9 Lo cierto es que a falta de evidencias claras sobre bosón de Higgs -introducido con el exclusivo propósito de renormalizar la teoría electrodébil- las reticencias de Dirac aparentan mayor justificación. Especialmente si pensamos que la existencia del bosón de Higgs conduce a valores de la constante cosmológica en flagrante contradicción con los datos observables. Véase Harari (1983) y Weinberg $(1987,1989)$ para esta interesante controversia. No obstante, la cromodinámica cuántica sí admite renormalización mediante el formalismo reticular. 
utilicen matemáticas inobjetables; no es inusual que elijan atajos dudosos para sus cálculos. [...] [P] ero en el caso de la teoría de renormalización nos las habemos con una teoría que ha salido victoriosa de todos los intentos realizados por los matemáticos para sanearla. Yo me inclino a pensar que la teoría de la renormalización no pervivirá mucho tiempo y que su notable concordancia con los experimentos ha de considerarse pura causalidad. (Dirac 1997, p. 8).

\section{EL PROBLEMA DE LA INTERACCIÓN}

Dejando a un lado las complicadas relaciones de las teorías cuánticas con sus contrapartidas clásicas (la física cuántica necesita un sustrato clásico susceptible de ser cuantizado, y también de suministrar una interpretación inteligible para los resultados experimentales), la teoría cuántica de campos parece ser inconsistente cuando se considera como una estructura lógica cerrada, incluso aunque su formalismo matemático resultase coherente, cosa que no sucede. La raíz de la dificultad estriba en idea subyacente de que los fenómenos físicos pueden ser diseccionados sin ambigüedad en componentes analizables por separado.

Así ocurre que la electrodinámica cuántica describe las interacciones considerando inicialmente ambos campos, electrónico y electromagnético, como entidades bien separadas, los detalles de cuya influencia mutua dependen críticamente de la situación específica en la cual dicha interacción se produzca. Una vez se conozcan esos detalles específicos, el modelo se construye partiendo del marco matemático general proporcionado por la teoría. Pero siempre subyace la idea de campos distintos en interacción, no de un único campo que se manifieste como materia o radiación según la perspectiva.

En el lenguaje lagrangiano, cuando un electrón interactúa con un campo electromagnético externo la descripción se realiza por medio de una suma de términos $L_{0}\left(\mathrm{~A}_{\mu}\right)+L_{0}(\psi)+L\left(\mathrm{~A}_{\mu}, \psi\right)$. Es obvio que $L_{0}\left(\mathrm{~A}_{\mu}\right)$ y $L_{0}(\psi)$ representan respectivamente los campos libres de Maxwell y de Dirac, en tanto que $L\left(\mathrm{~A}_{\mu}\right.$, $\psi)$ es el término de interacción. La forma de este último se obtiene imponiendo condiciones de invariancia relativista, de simplicidad formal y de correspondencia con la teoría clásica de Lorentz sobre los electrones. En general, $L\left(\mathrm{~A}_{\mu}\right.$, $\psi) \equiv e \psi^{*} \gamma^{\mu} \psi \mathrm{A}_{\mu}$ donde $e$ es la carga del electrón y $\gamma^{\mu}$ una matriz de Dirac. Se observa, pues, que de principio a fin la electrodinámica cuántica, como teoría de las interacciones entre materia y radiación, presenta los campos que interactúan diáfanamente separados. En la densidad lagrangiana tenemos un término para cada campo $\left(\mathrm{A}_{\mu} \mathrm{y} \psi\right)$ y un término de acoplamiento para la interacción entre ambos.

El problema con el cual nos topamos ahora radica en la imposibilidad de aplicar los espacios de Fock -diseñados para campos libres- a los procesos de 
interacción. La descomposición de las ondas clásicas en sus componentes de Fourier convenientemente cuantizados, permite introducir el concepto de "cuanto" en la teoría. Semejante proceder se torna imposible en el caso de campos interactuantes; simplemente no existe un desarrollo de Fourier adecuado para ello, puesto que carecemos de un espacio de Fock para campos en interacción. Las consecuencias resultan dramáticas: la propia idea de que un estado de interacción entre campos pueda describirse como una distribución de probabilidad sobre configuraciones clásicas de dichos campos, queda ciertamente en entredicho. ${ }^{10}$ De acuerdo con el teorema de Haag (1955) no existe una transformación unitaria que relacione los operadores hamiltonianos correspondientes al caso de los campos libres con el de la interacción entre ellos. Es cierto que las técnicas de renormalización ofrecen la posibilidad de suspender la aplicación del teorema de Haag. En efecto, al introducir términos compensatorios infinitos, el formalismo matemático no queda bien definido.

La cuestión presenta un aspecto todavía más confuso si pensamos que los desarrollos perturbativos -indispensables en la electrodinámica cuántica- no son meramente herramientas matemáticas destinadas a facilitar los cálculos. Bien al contrario, lo cierto es que no existen ecuaciones exactas cuyas aproximaciones nos vengan dadas por los desarrollos perturbativos; el propio cálculo de perturbaciones constituye el núcleo duro de la teoría y sus modelos, si es que podemos distinguir legítimamente entre la una y los otros. Sin desarrollos perturbativos de la matriz $S$, por ejemplo, careceríamos de predicciones y resultados que comparar con los datos experimentales. Ahora bien, el uso de los métodos perturbativos en la electrodinámica cuántica se sustenta sobre la presunta debilidad de la interacción entre los campos de Dirac y de Maxwell, suposición que muy a menudo se halla lejos de estar garantizada. ${ }^{11}$

En la práctica, sólo manejamos los primeros términos del desarrollo perturbativo de la matriz de dispersión asociada al caso concreto que nos ocupa, sin considerar los campos como un sistema global en interacción. La matriz $S$ "exacta" simplemente no existe. Y en el mejor de los casos tomamos unos pocos términos más del desarrollo, representativos de diversas formas de interacción entre cuantones, dejando que los resultados experimentales decidan su pertinencia. Podría parecer que las integrales de camino de Feynman servirían para evadir este atolladero, pero de nuevo nuestras esperanzas se ven frustradas. En los modelos concretos donde tales integrales se aplican, las amplitudes de transición no se pueden desarrollar en series infinitas en las que aparezcan los propagadores de los cuantos. Por ello, la interpretación habitual que las asocia

10 Interesantes discusiones sobre estos argumentos, que en algunos casos llegan a cuestionar los conceptos de "cuantón" y "campo" a la vez, se hallan en Earman y Fraser (2006), p. 330.

11 Véase un agudo comentario al respecto en Mandl y Shaw (1984), p. 95. 
con cuantones no es susceptible de traducirse en un desarrollo finito basado en integrales de camino (Rueger 1992, p. 314).

Tampoco ha permanecido ajeno al debate el carácter unitario de la matriz $S$ para los casos realistas de campos cuánticos en interacción. Ni se ha garantizado su cumplimiento en el espacio-tiempo de Minkowski, ni menos aún en una variedad curva. Y no es una cuestión menor, pues se admite tácitamente que la naturaleza unitaria de la matriz $S$ se da como consecuencia de la conservación de la probabilidad en el tiempo. Dicha conservación recoge el hecho de que la evolución temporal de un sistema cuántico gobernada por la ecuación de Schroedinger, se representa mediante un conjunto de operadores unitarios que, como tales, conservan las probabilidades de transición entre distintos estados. Sin embargo, sirviéndose de modelos muy idealizados (Kato y Kuroda 1959), y posteriormente con situaciones mucho más plausibles y realistas, ${ }^{12}$ se han expuestos ejemplos teóricos en los que no siempre habría de suceder necesariamente así.

El ruso Lev Landau expresó su convicción de que las únicas cantidades físicas directamente observables eran las asociadas con las partículas asintóticamente libres, como sus impulsos o energías antes y después de la dispersión (Cao y Schweber 1993, p. 47). La idea consistía en dejar de lado el acto mismo de la interacción -demasiado complejo para un tratamiento detallado- para concentrarse en los estados iniciales y finales del proceso. En consecuencia, Landau sostenía que los campos cuánticos interpolados entre ambos estados límites (partículas infinitamente alejadas antes y después de la interacción) carecían de significado físico, y abogaba por una ruptura con la teoría cuántica de campos a favor de una aproximación semejante a la matriz $S$ de Heisenberg.

El uso de los espacios de Fock para representar los sistemas sin interacción, sustenta la interpretación cuántica -discreta, discontinua- de los campos de fuerzas como un agregado de bosones (fotones, en el caso electromagnético). Nada de eso, empero, nos garantiza que pueda obrarse igual cuando hay interacciones presentes, porque entonces las representaciones de Fock no resultan una herramienta adecuada.

La respuesta obvia sería ahora generalizar el concepto de espacio de Fock de manera que abarcase también los casos de interacción, si bien todos los intentos llevados a cabo en esa dirección han resultado infructuosos. La representación de un sistema cuántico mediante espacios de Hilbert no puede lograrse con una

12 El modelo de Pearson (1975) emplea un potencial local oscilante que varía más rápidamente según nos acercamos al origen del sistema de referencia, situado en su centro de masas. Así, cuando una partícula cuántica ingresa en el sistema en un estado asintóticamente libre, queda atrapada para siempre en ese potencial, y no genera un conjunto de estados de salida asintóticamente libres. 
mera descomposición de Fourier de campos clásicos en interacción, porque las expresiones obtenidas no serían covariantes relativistas y por ello quedarían automáticamente excluidas como candidatas para una buena teoría de campos cuánticos. Las referencias habituales en la literatura especializada, suelen imputar este problema a la dificultad de representar estados con dos o más partículas en el marco de los espacios de Fock (Bain 2000, p. 393).

Es cierto que en un sistema sin interacción la energía de un estado con dos partículas es simplemente la suma de las energías de los dos estados con una sola partícula, en tanto que la presencia de interacciones introduce términos adicionales que oscurecen enormemente la situación. No obstante, un examen más detenido revela que esa no es la fuente principal de nuestro dilema, puesto que también aparece en los estados de vacío, los cuales carecen por definición de partículas "reales" (dejamos a un lado las "virtuales", desde luego). Los responsables de todo ello parece ser los requisitos relativistas impuestos a la teoría cuántica (Earman y Fraser 2006, sec. 4), pues el teorema de Haag descansa sobre premisas relativistas, mientras que ni la descomposición de Fourier ni los estados sin partículas en la representación de Fock son covariantes bajo el grupo de Poincaré. Como consecuencia, la relatividad especial no puede asignar correctamente las energías a los estados de una partícula, cosa que sí ocurre en los sistemas libres, donde las ecuaciones son lineales y la relatividad permite una interpretación cuantizada de los campos. Cuando tenemos campos interactuantes, el encaje de ecuaciones de campo no lineales con las exigencias relativistas impide una tal interpretación, y toda mención a cuantos mediadores de interacciones permanece injustificada.

\section{Más ALLÁ de LA TeORÍA CuÁNTICA de CAMPos}

A quienes conciben el realismo como la única posición seriamente sostenible en la filosofía de la ciencia, se les confronta a menudo con la paradójica circunstancia de que la teoría cuyas predicciones se satisfacen con mayor precisión, funda esos mismos resultados en una manipulación poco escrupulosa de los términos divergentes de orden superior en los desarrollos perturbativos que permiten calcular las soluciones aproximadas de la teoría (Weinberg 1977, Weisskopf 1981, Teller 1988). Esta incongruencia se suele atribuir o bien a la drástica diferencia de planteamiento entre la física cuántica y la relativista (disparidad que acaso se deba al carácter incompleto de ambas), o bien a la insuficiencia del arsenal matemático disponible para describir un ámbito de la realidad -el que la teoría cuántica de campos pretende reflejar- radicalmente distinto del que nos es familiar. Expresado con mayor elegancia (Rohrlich, 1996, p. 94): «[...] a veces es difícil decir si un desacuerdo con el experimento se debe a la forma 
aproximada en que se resuelven las ecuaciones o si el desacuerdo se debe a las ecuaciones fundamentales de la teoría misma».

Haciendo de la necesidad virtud, los teóricos emplean la posibilidad de renormalización como un filtro a través del cual seleccionar las teorías presuntamente más prometedoras. Aquellas que no resulten renormalizables se desechan sin rodeos, con independencia del resto de sus méritos; y en verdad es un poderoso criterio de selección. Como también es cierto que, si admitimos la existencia de una teoría libre de divergencias y aún desconocida de la cual los campos cuánticos son meras aproximaciones, entonces surge la siguiente cuestión:

¿Cómo, cabría preguntase, puede la renormalizabilidad, entendida de este modo, funcionar como una restricción sobre la construcción de teorías? Recordemos que la renormalizabilidad era el requisito de que todos los términos divergentes pudiesen absorberse en un número finito de constantes observables. El requerimiento de que una teoría tenga esta propiedad estrecha severamente el abanico de opciones permitido por otras restricciones, como la invariancia de Lorentz y la de gauge. ¿Pero cómo hemos de justificar el requisito de renormalizabilidad en tanto que restricción que una teoría aproximada debe satisfacer? Si [...] la teoría correcta no tiene términos divergentes, la restricción (que todos los términos divergentes sean absorbibles) no puede justificarse sobre la base de que una teoría verdadera (o todas las teorías más precisas) debe satisfacer la restricción. Así que, ¿por qué deberíamos creer que esta restricción nos guiará hacia teorías mejores? (Teller, 1995, p. 167)

Avanzando sólo un paso más, la pregunta podría ser si hay razones para suponer que las consecuencias de una renormalización perturbativa se aproximan a las de una teoría cuántica de campos exacta (Huggett 2000, p. 630), o incluso si existe tal cosa como una teoría cuántica de campos "exacta". La respuesta -suele afirmarse - reside en la teoría de grupos de renormalización (Huggett y Weingard 1995), gracias a la cual las divergencias pueden interpretarse como producto de una elección incorrecta del formalismo, compensada cuando las técnicas de renormalización "afinan" - por así decirlo- los parámetros de los campos cuánticos.

Llegamos con ello a una de las claves de la controversia, porque se hace imperativo reconocer que a pesar de sus incongruencias la teoría cuántica de campos suministra predicciones de formidable exactitud. Por imaginarlo gráficamente, supongamos que, en efecto, existe una correspondencia entre el conjunto de las teorías cuánticas de campos y las propiedades que caracterizan los sistemas de objetos cuánticos. En su forma actual esta correspondencia conduce a valores infinitos, las temidas divergencias de la teoría. Pero se da 
la circunstancia de que tenemos recetas extrateóricas -las técnicas de renormalización- que desvían esa correspondencia hacia un conjunto de valores extremadamente ajustados a los datos empíricos.

El dilema estriba en si hemos de considerar esas recetas suplementarias como una pieza más de la teoría -y una pieza esencial-o deberíamos buscar otra correspondencia que nos condujese a los valores finitos adecuados sin necesidad de reajustes sobrevenidos. No es una cuestión baladí, en tanto la rigidez formal de las teorías cuánticas de campos hace muy difícil cualquier tipo de retoque o modificación siquiera parcial sin amenazar con la ruina de todo el edificio. La alternativa, innecesario es decirlo, encierra todavía mayores complicaciones, pues consistiría, ni más ni menos, que en la búsqueda de una teoría capaz de sustituir desde su origen todo el programa unificador de las teorías cuánticas de campos. Esta nueva construcción habría de realizarse sobre bases conceptuales dramáticamente distintas, si desea tener esperanzas de éxito donde sus predecesoras fracasaron, conservando a la vez el impresionante repertorio de éxitos de estas últimas. No es de extrañar que de momento nadie haya emprendido un proyecto semejante.

La búsqueda de un reemplazo se ve dificultada por el hecho de que la teoría cuántica de campos -igual que en la teoría cuántica sin campos-cuenta con diversas formulaciones matemática y empíricamente equivalentes; por ejemplo, mediante espacios de Hilbert o a través de las integrales de camino de Feynman. Y esta variedad de planteamientos tal vez deba contemplarse como un reto para quien pretenda extraer conclusiones ontológicas de los campos cuánticos (Rohrlich 1996).

\section{Ontología}

La teoría cuántica de campos, que aspira a establecer el marco general en el que insertar los fundamentos de la física, se ve obligada a afrontar cuestiones muy profundas sobre los constituyentes últimos del universo. ¿De qué clase son las entidades elementales que configuran el mundo material?; ¿corpúsculos, campos o algo distinto de ambos? Más aún, ¿tiene sentido preguntarse por unos entes básicos con los cuales construir la realidad física? Si consideramos que la individualidad es un ingrediente indispensable de las partículas, el principio cuántico de indiscernibilidad y la variabilidad en el número de partículas de los sistemas interaccionantes, tornan problemática semejante adscripción (Teller 1995, Seibt 2002).

Además, el hecho de que los campos sean sistemas con infinitos grados de libertad, en abierta oposición con el concepto de partícula -con un número finito de grados de libertad-complica aún más las cosas. Es cierto que Wigner 
identificó las diversas clases de partículas elementales con representaciones unitarias irreducibles del grupo de Poincaré (Wigner 1939, Newton y Wigner 1949, Buchholz 1994), pero esto tan solo supone una clasificación de tales entidades, no una definición de las mismas. La idea consiste en que, si la teoría cuántica relativista puede interpretarse en términos de partículas, entonces el catálogo de posibles partículas viene dado por las representaciones unitarias irreducibles del grupo de Poincaré. La legitimidad última de una interpretación corpuscular tal, no entra en discusión.

Bajo los requerimientos de la relatividad especial la condición de localizabilidad queda en entredicho, pues la teoría excluye la posibilidad de ubicar una partícula cuántica en cualquier región espacial de tamaño finito. Y en relación con ello se han estipulado teoremas que impiden distinguir entre el estado de vacío y un sistema con un número específico de partículas mediante medidas puramente locales (Reeh y Schlieder 1961, Redhead 1995, Clifton y Halvorson 2001). Peor aún, otro teorema prescribe que una teoría cuántico-relativista con un número fijo de partículas, obtendrá siempre una probabilidad nula de localizar una de tales partículas en cualquier región espacial finita ${ }^{13}$ (Borchers 1967, Saunders 1995, Malament 1996, Busch 1999, Fleming y Butterfield 1999, Clifton y Halvorson 2002).

Todavía más desafiante para la ontología corpuscular de los campos cuánticos, fue el descubrimiento de que un observador uniformemente acelerado en un espacio-tiempo vacío de Minkowski -sin gravitación- detectará partículas con una distribución térmica de energías (Unruh 1976, Unruh y Wald 1984). Esta conclusión evidencia el carácter dependiente del observador del concepto de partícula, incluso en un espacio-tiempo sin curvatura. ${ }^{14}$ No obstante, tampoco cabe negar que el carácter discontinuo de ciertas propiedades, como la carga eléctrica, parece abogar por la existencia de entidades discretas en algún sentido. Sin olvidar que las contrastaciones experimentales de la teoría cuántica de campos se consiguen acelerando y provocando colisiones entre lo que parecen ser partículas.

Si admitimos el campo cuántico como entidad básica de la realidad física, a diferencia de los campos clásicos, no cabe asignar un número real (representativo del valor de una magnitud física) a cada punto del espacio-tiempo, sino un operador cuántico que representa el abanico completo de todos los valores posibles de una magnitud física. Esto es algo muy distinto de la típica noción

13 Este resultado presupone el cumplimiento de cuatro condiciones: covariancia relativista, conservación de la energía, localizabilidad y localidad.

14 La existencia de un operador asociado con el número de partículas parece depender del grupo de simetría de Poincaré, que se pierde en un espacio-tiempo curvo como el de la relatividad general. 
de campo físico, lo que despierta legítimas dudas sobre su pertinencia (Teller 1990, Teller 1995).

Tan abrupta parece la senda hacia partículas y campos que no han faltado propuestas favorables a otras ontologías. Desde los puros sucesos espacio-temporales (Auyang 1995, Dieks 2002) o los procesos físicos (Seibt 2002, Hättich 2004, Kuhlmann 2000, Kuhlmann 2002) hasta rasgos estructurales abstractos pero considerados objetivos, como las simetrías (Cao 1997b, Ladyman 1998, Brading y Castellani 2003).

A mi juicio, la ontología no es un lujo del cual puedan prescindir las teorías físicas que aspiran a un carácter fundamental. No son ociosos, pues, los esfuerzos realizados a lo largo de los años por dotar a la física cuántica con una ontología coherente, y sus escollos tan solo indican que quizás debamos buscar en otra dirección. Al fin y al cabo las nociones de corpúsculo y campo vienen ligadas al nivel macroscópico como idealización de los objetos de nuestra experiencia. Y la teoría cuántica de campos representa la tentativa de obtener un híbrido fértil de la unión entre ambas. Pero el mundo cuántico difiere de nuestras expectativas empíricas usuales, apunta a una realidad distinta y sin duda precisa nuevos objetos básicos para su ontología. Necesitamos un nuevo elemento fundamental -o varios- cuyas características permitan elaborar una ontología común para el espacio-tiempo, la materia y sus interacciones. Los fracasados intentos de reducir la materia a relaciones espacio-temporales y viceversa, sugieren poderosamente que podría existir un sustrato subyacente, una ontología compartida de cuya riqueza fenomenológica las perplejidades cuánticas son una manifestación.

\section{CONCLUSIONES}

El cuadro general de la situación se muestra tan enrevesado que numerosos autores han acabado manifestando los mismos sentimientos que se recogen en la siguiente cita:

Cuando se contempla la sucesión de alteraciones ad hoc realizadas en la teoría cuántica de campos (mar de electrones de energía negativa, desprecio de autoenergías infinitas y polarizaciones del vacío, invariancia gauge local, renormalización forzada en teorías gauge, ruptura espontánea de simetría, confinamiento de los quarks, color, entre otros ejemplos) y la imagen que emerge del "vacío" (¿éter?), borboteando con pares partícula-antipartícula de todas clases y responsable de la ruptura de las simetrías inicialmente presentes, uno puede preguntar si se supone seriamente o no que la naturaleza ha de ser así. (Cushing 1982, p. 78 , cursivas en el original). 
La electrodinámica cuántica ha suministrado resultados demasiado buenos para que pensemos en desprendernos de ella sin contar con un recambio al menos igual de satisfactorio. Un recambio -no lo olvidemos- que logre combinar la teoría cuántica con, al menos, la relatividad especial de Einstein. Ahí se halla el nudo de la cuestión, puesto que numerosos textos dan por conseguido este objetivo sin que haya plena garantía de ello.

Es un recurso común mencionar el hecho de que las teorías cuánticas de campos se dan en forma lagrangiana para lograr una equiparación entre las variables de posición y la de tiempo. Así, la densidad lagrangiana $\mathcal{L}$ se juzga relativista si depende de un 4-vector de posición espacio-temporal, $x_{\mu}$, sometido al grupo de Poincaré. Pero tal vez no sea esa la denominación más adecuada. Quizás bastase con llamar a tales magnitudes "covariantes de Poincaré", porque de hecho es lo que son, y no "relativistas". La experiencia nos ha enseñado que las dos teorías relativistas de Einstein exhiben una característica que, a falta de mejor nombre, cabría llamar "geometrizabilidad". El espacio-tiempo minkowskiano de la Relatividad Especial posee una métrica típica, que determina el comportamiento de los campos que sobre él se despliegan (por ejemplo, el electromagnético) y, en general, el tipo de procesos físicos posibles en su seno. Y no digamos de la Relatividad General, donde la misma gravitación se incorpora a la estructura geométrica de un espacio-tiempo pseudo-riemanniano. Estas dos descripciones de la realidad son posibles en tanto que tratemos con funciones continuas que asignen a cada punto del espacio-tiempo una propiedad física individualizable y causalmente separable. ${ }^{15}$

Sin embargo, cuando accedemos a la teoría cuántica de campos, la situación es muy distinta. No tenemos funciones continuas del espacio y el tiempo, sino distribuciones de operadores que nos remiten a un espacio abstracto de Hilbert (espacio de estados, espacio de Fock, etc.) el cual se adopta como verdadero escenario de los procesos físicos. Dado que la vinculación entre tales espacios abstractos y el espacio-tiempo físico resulta indirecta, remota y enteramente abierta a la controversia, no parece aconsejable considerar relativista una densidad lagrangiana por el mero hecho de que sus coordenadas de posición y tiempo cumplan las simetrías de Poincaré. Nada nos impide, desde luego, considerar la geometrizabilidad un rasgo accesorio de la teoría, y quedarnos tan solo con la obediencia del grupo de Poincaré como criterio decisivo de su índole relativista. Pero no podemos entonces ahuyentar la sensación de que algo se pierde con semejante proceder, como acaso demuestra el hecho de que un siglo después de la Relatividad General no se haya logrado cuantizar la gravedad.

En el fondo nos las vemos con un problema que aquejó ya la teoría cuántica

15 Entendido en el habitual sentido relativista: dos puntos separados por un intervalo de tipo espacial no guardan entre sí relación causal alguna. 
original y se transmitió intacto a la de campos. La interpretación física de las coordenadas $x_{\mu}$ en las teorías cuánticas, difiere del resto de la física, ya que los cuantones carecen de propiedades clásicas como la de "posición”. Esta carencia se traslada igualmente a la teoría cuántica de campos, amplificada por su colisión frontal con la idea clásica de campo como un sistema continuo con infinitos grados de libertad. La consecuencia es la multiplicación infinita de la falta de noción de posición en los cuantones, hasta un grado en el cual la propia noción de cuantos de campo la hace controvertida:

Los modos normales, los cuantos del campo, y las partículas son buenos conceptos para describir los sistemas continuos sólo cuando el acoplamiento entre ellos es despreciable. La condición no siempre se satisface. Por ejemplo, los modos de una cuerda de violín no pueden considerarse independientes unos de otros cuando la vibración es bastante violenta para hacerse inarmónica. Igualmente, cuando los campos cuánticos interactúan, los cuantos pueden excitarse y desexcitarse fácilmente de modo que la imagen estática de los campos cuánticos trazada más arriba ya no se aplica. Por ello los teóricos de los campos dicen que las partículas son epifenómenos y el concepto de partícula no es central en la descripción de los campos. (Auyang 1995, p. 53).

La electrodinámica cuántica parece involucrar problemas de una índole muy particular. Bien podría suceder que sea necesaria una nueva teoría de interacciones fundamentales que combine desde su mismo origen los requerimientos cuánticos y relativistas. En ese sentido el propio nombre de la teoría resulta cuando menos poco adecuado. Hablar de "campos cuánticos" evoca a la vez la idea de interacción local heredada de la física clásica y el carácter discreto -indigerible para el campo tradicional- propio de la física cuántica. Tal vez hubiese sido más oportuno referirse a ella como la "teoría gauge con invariancia de Poincaré para sistemas cuánticos con $n$ componentes libres o débilmente interaccionantes"; pero sin duda hubiera sido también mucho menos atractivo.

Sin embargo, a la luz de cuanto se ha expuesto una serie de consideraciones sí parecen ineludibles. En primer lugar, todo apunta a que haríamos bien en buscar un nuevo tipo de descripción para los componentes fundamentales de la naturaleza. Si el modelo corpuscular de los quarks se queda corto, cuarenta años de teoría de supercuerdas tampoco han acarreado un genuino progreso en nuestro conocimiento de la intimidad de la materia. Y las investigaciones sobre objetos como las $p$-branas, sustentadas en un puro virtuosismo matemático comienzan a entrar en declive en tanto que teorías físicas. ¿Qué nos espera tras el siguiente recodo del camino? Resulta imposible atisbarlo.

La infructuosidad de los esfuerzos realizados hasta la fecha sugiere no 
sólo un replanteamiento de nuestros presupuestos ontológicos sobre la materia, sino también sobre el propio espacio-tiempo. Nada inverosímil es suponer que necesitamos conceptos nuevos sobre los cuales construir las nociones de materia e interacción junto con las de espacio y tiempo. La participación del espaciotiempo en la interacción gravitatoria, revelada por la relatividad general, puede ser el primer paso en un camino aún apenas recorrido.

¿Y qué puede ser más fascinante para un físico -o para un filósofo- que la existencia de elementos previos al espacio y al tiempo? Ante un desafío semejante nuestra imaginación científica puede verse empujada hasta el límite de sus fuerzas. Pues, como una paradoja más de la naturaleza, en nuestra capacidad imaginativa descansa la posibilidad de desvelar la realidad física.

\section{REFERENCIAS}

AUYANG, S. Y. 1995: How is quantum theory of fields possible? New York: Oxford Univ. Press.

BAIN, J. 2000: «Against particle/field duality: asymptotic particle states and interpolating fields in interacting QFT (Or: Who's afraid of Haag's theorem?)», Erkenntnis, 53, pp. 375-406.

BEREZIN. F. A. 1966: The Method of Second Quantization. New York: Academic Press.

BOGOLIUBOV, N. N., SHIRKOV, D. V. 1959: Introduction to the theory of quantized fields. New York: Interscience Publishers.

BOGOLIUBOV, N. N., LOGUNOV, A. A., TODOROV, I. T. 1975: Introduction to Axiomatic Quantum Field Theory. Reading (Mass.): Benjamin.

BORCHERS, H. J. 1967: «A Remark on Theorem by B. Misra», Communications in Mathematical Physics, 4, pp. 315-323.

BRADING, K., CASTELLANI, E. (eds.) 2003: Symmetries in Physics: Philosophical Reflections. Cambridge: Cambridge University Press.

BUCHHOLZ, D. 1998: «Current trends in axiomatic qantum field theory», en P. Breitenlohner y D. Maison (eds.), Quantum Field Theory. Proceedings of the Ringberg Workshop Held at Tegernsee, Germany, 21-24 June 1998 On the Occasion of Wolfhart Zimmermann's 70th Birthday (Lecture Notes in Physics vol. 558). Berlin-Heidelberg: Springer, pp. 43-64.

BUSCH, P. 1999: «Unsharp localization and causality in relativistic quantum theory», Journal of Physics A: Mathematics General, 32, 6535.

CAO, T.Y., SCHWEBER, S. S. 1993: «The conceptual foundations and philosophical aspects of renormalization theory», Synthese, 97, pp. 33-108.

CAO, T.Y. 1997: Conceptual development of 20th century field theory. Cambridge: Cambridge University Press.

CAO, T. Y. 1997a: Conceptual Developments of 20th Century Field Theories. Cambridge: Cambridge University Press.

CAO, T. Y., 1997b: Introduction: Conceptual issues in QFT, en Cao 1997a, pp. 1-27. 
CAO, T. Y. (ed.) 1999: Conceptual Foundations of Quantum Field Theories. Cambridge: Cambridge University Press.

ClAdÉ, P., DE MIRANDES, E., CADORET, M., GUELlATI-KHÉLIFA, S., SCHWOB, C., NEZ, F., JULIEN, L., BIRABEN, F. 2006: «Determination of the fine structure constant based on Bloch oscillations of ultracold atoms in a vertical optical lattice», Phys. Rev. Lett., 96, 033001.

CLIFTON, R., HALVORSON, H. 2001: «Entanglement and open systems in algebraic quantum field theory», Studies in History and Philosophy of Modern Physics, 32, pp. 1-31.

CLIFTON, R., HALVORSON, H. 2002: «No place for particles in relativistic quantum theories? », Philosophy of Science, 69, pp. 1-28.

CUSHING, J. T. 1982: «Models and Methodologies in Current High-Energy Physics», Synthese, 50, pp. 5-101.

CUSHING, J. T. 1990: Theory Construction and Selection in Modern Physics: The $S$-Matrix. Cambridge: Cambridge University Press.

DIEKS, D. 2002: «Events and covariance in the interpretation of quantum field theory», en Kuhlmann et al. 2002, pp. 215-234.

DIRAC, P. A. M. 1978: en H. Hora, J. R. Shepanski (eds.) Directions in Physics, New York: Wiley.

DIRAC, P. A. M. 1987: en B. N. Kursunoglu, E. P. Wigner (eds.) Reminiscences About a Great Physicist: Paul Adrien Maurice Dirac, Cambridge: Cam. Univ. Press.

DIRAC, P. A. M. 1997: «La concepción física de la naturaleza», en Misterios de la física cuántica, Temas de Investigación y Ciencia (Barcelona: Prensa Científica S.A.), vol. 10, pp. 1-8.

DYSON, F. J. 1952: «Divergence of Perturbation Theory in Quantum Electrodynamics», Phys. Rev., 85, pp. 631-632.

EARMAN, J., FRASER, D. 2006: «Haag's theorem and its implications for the foundations of quantum field theory», Erkenntnis, 64, pp. 305-344.

EPSTEIN, S. T. 1948: «Perturbation Theory and Its Applications in Quantum Field Theory» Phys. Rev., 73, pp. 179-183.

FADDEEV, L. D., SLAVNOV, A. A. 1991: Gauge Fields: An Introduction to Quantum Theory. New York: Addison-Wesley.

FEYNMAN, R. P. 1948: «Relativistic cut-off for quantum electrodynamics», Phys. Rev., 74, pp. 1430-1438.

FEYNMAN, R. P. 1949: «Space-time approach to quantum electrodynamics», Phys. Rev., 76, pp. 769-789.

FEYNMAN, R. P. 1962: The theory of fundamental processes. Reading (Mass.): W. A. Benjamin Inc.,

FLEMING, G. N., BUTTERFIELD, J. 1999: «Strange positions», en J. Butterfield, C. Pagonis (eds.), From Physics to Philosophy, Cambridge: Cambridge University Press, pp. 108-165.

GABRIELSE, G., HANNEKE, D., KINOSHITA, T., NIO, M., ODOM, B. 2006: «New Determination of the Fine Structure Constant from the Electron $g$ Value and QED», Phys. Rev. Lett., 97, 030802. 
El significado filosófico de la teoría cuántica de campos

GEL'FAND, I. M., SHILOV, G. E. 1964: Generalized Functions, vol. I. New York: Academic Press.

GEL'FAND, I. M., VILENKIN, N. 1968: Generalized Functions, vol. IV. New York: Academic Press.

HAAG, R. 1955: «On quantum field theories», Det Kongelige Danske Videnskabernes Selskab, Matematisk-fysiske Meddelelser, 29/12, pp. 1-37.

HAGIWARA, K., MARTIN, A.D., NOMURA, D., TEUBNER, T. 2007: «Improved predictions for $\mathrm{g}-2$ of the muon and QED(M2Z)», Phys. Lett. B, 649, pp. 173185.

HARARI, H. 1983: «The structure of quarks and leptons», Sci. Am., 248, IV, 56.

HARDY, G. H. 1949: Divergent Series. London: Oxford University Press.

HÄTTICH, F. 2004: Quantum Processes. A Whiteheadian Interpretation of Quantum Field Theory. Münster: Agenda Verlag.

HAUGAN, M. P., WILL, C. M. 1989: «Testing Local Lorentz Invariance using laboratory and space technology», Advances in Space Research, 9, pp. 133-137.

HEITLER, W. 1954: The Quantum Theory of Radiation. Oxford: Clarendon Press.

HUGGETT, N. 2000: «Philosophical Foundations of Quantum Field Theory», British J. Phil. Sci., 51, pp. 617-637.

HUGGETT, N., WEINGARD, R. 1995: «The Renormalization Group and the Effective Field Theory Programme», Synthese 102, pp. 171-194.

ITZYKSON, C., ZUBER, J.-B. 1980: Quantum Field Theory. New York: McGrawHill.

JOST, R. 1965: The General Theory of Quantized Fields. Providence: American Mathematical Society.

KARSHENBOIM, S.G. 2005: «Fundamental physical constants: their role in physics and metrology and recommended values», Physics-Uspekhi, 48, 255.

KARSHENBOIM S.G., FENDEL, P., IVANOV, V.G., KOLACHEVSKY N., HÄNSCH, T.W. 2005: «Study of hyperfine structure in simple atoms and precision tests of the bound state QED», Can. J. Phys., 83, pp. 260-263.

KATO, T., KURODA, S.T. 1959: «A remark on the unitary property of the scattering operator», Nuovo Cimento, 14, pp. 1102-1107.

KRISCH, A. D. 1987: «An experimenter's view of P.A.M. Dirac», en B. N. Kursunoglu \& E. P. Wigner (eds.), Reminiscences About a Great Physicist: Paul Adrien Maurice Dirac, Cambridge: Cam. Univ. Press.

KUHLMANN, M. 2000: «Processes as objects of quantum field theory», en J. Faye, U. Scheffler, M. Urchs (eds.), Events, Facts and Things. Poznan: Poznan Studies in the Philosophy of the Sciences and the Humanities, pp. 365-388.

KUHLMANN, M., 2002: «Analytical ontologists in action: A comment on Seibt and Simons», en Kuhlmann et al. 2002, pp. 99-109.

KUHLMANN, M., LYRE, H., WAYNE, A. (eds.) 2002: Ontological Aspects of Quantum Field Theory. Singapore-London-Hackensack: World Scientific Publishing Company.

LADYMAN, J. 1998: «What is structural realism?», Studies in History and Philosophy of Science, 29, pp. 409-424. 
LEE, R. N., MILSTEIN, A. I., TEREKHOV, I. S., KARSHENBOIM, S. G. 2005: «Virtual light-by-light scattering and the $\mathrm{g}$ factor of a bound electron», Phys. Rev. A, 71, 052501.

LEWIS, H. W. 1948: «On the Reactive Terms in Quantum Electrodynamics», Phys. Rev., 73, p. 173.

MALAMENT, D. 1996: «In defense of dogma: Why there cannot be a relativistic quantum mechanics of (localizable) particles», en R. Clifton (ed.), Perspectives on Quantum Reality. Kluwer: Academic Press, pp. 1-10.

MANDL, F., SHAW, G. 1984: Quantum field theory. New York: Wiley.

NEWTON, T. D., WIGNER, E. P. 1949: «Localized states for elementary particles», Reviews of Modern Physics, 21/3, pp. 400-406.

ODOM, B., HANNEKE, D., D’URSO, B., GABRIELSE, G., 2006: «New Measurement of the Electron Magnetic Moment Using a One-Electron Quantum Cyclotron», Phys. Rev. Lett., 97, 030801.

PACHUCKI, K., CZARNECKI, A., JENTSCHURA, U. D., YEROKHIN, V. A. 2005: «Complete two-loop correction to the bound-electron g factor», Phys. Rev., 72, 022108.

PAIS, A. 1986: Inward Bound. Of Matter and Forces in the Physical World. Oxford - New York: Clarendon \& Oxford University Press.

PEARSON, D. 1975: «Quaternionic quantum field theory», Commun. Math. Phys., 40, pp. $125-131$.

PENROSE, R. 2006: El camino a la realidad. Madrid: Debate

REDHEAD, M. L. G. 1995: «More ado about nothing», Foundations of Physics, 25 , pp. 123-137.

REEH, H., SCHLIEDER, S. 1961: «Bemerkungen zur Unitäräquivalenz von Lorentzinvarianten Feldern», Nuovo Cimento, 22, pp. 1051-1068.

ROHRLICH, F. 1996: «Interpreting Quantum Field Theory», Stud. Hist. Phil. Mod. Phys., 27, pp. 91-98.

RUEGER, A. 1992: «Attitudes towards infinities: Responses to anomalies in quantum electrodynamics, 1927-1947», Historical Studies in the Physical and Biological Sciences, 22, pp. 309-337.

SAUNDERS, S. 1995: «A dissolution of the problem of locality», en Hull, M. F. D., Forbes, M., Burian, R. M. (eds.), Proceedings of the Biennial Meeting of the Philosophy of Science Association: PSA 1994. East Lansing (MI): Philosophy of Science Association, vol. 2, pp. 88-98.

SEIBT, J. 2002: «The matrix of ontological thinking: Heuristic preliminaries for an ontology of QFT», en Kuhlmann et al. 2002, pp. 53-97.

SCHWINGER, J. (ed.) 1958: Quantum Electrodynamics. New York: Dover.

SICK, I. 2005: «Proton-polarizability effect in the Lamb shift for the hydrogen atom», Progr. Part. Nucl. Phys., 55, p. 440.

SOKAL, A. D. 1980: «An improvement of Watson's theorem on Borel summability», J. Math. Phys., 21, p. 261.

STREATER, R.F., WIGHTMAN, A.S. 1980: PCT, Spin and Statistics, and all That. Reading: Benjamin. 
TELLER, P. 1988: «The Problems of Renormalization», en H. R. Brown, R. Harré (eds.) Philosophical Foundations of Quantum Field Theory. Oxford: Clarendon Press, pp. 73-89.

TELLER, P. 1990: «What the quantum field is not», Philosophical Topics, 18, pp. 175-186.

TELLER, P. 1995: An Interpretive Introduction to Quantum Field Theory. Princeton: Princeton University Press.

UNRUH, W. G. 1976: «Notes on black hole evaporation», Physical Review D, 14, pp. 870-92.

UNRUH, W. G. WALD, R. M. 1984: «What happens when an accelerating observer detects a Rindler particle?», Physical Review D, 29, pp. 1047-1056.

WEINBERG, S. 1977: «The Search for Unity: Notes for a History of Quantum Field Theory», Daedalus, Fall Iss., pp. 17-35.

WEINBERG, S. 1987: «Particle Physics and Inflationary Cosmology», Phys. Today, 40, I, p. 7.

WEINBERG, S. 1989: «The cosmological constant problem», Rev. Mod. Phys., 61, p.1.

WEISSKOPF, V. F. 1981: «The development of field theory in the last 50 years», Physics Today, 3, pp. 358-373.

WIGNER, E.P. 1939: «On unitary representations of the inhomogeneous Lorentz group», Ann. Math., 40, pp. 149-204.

Rafael Andrés Alemañ Berenguer es miembro del Departamento de Ciencia de Materiales, Óptica y Tecnología Electrónica de la Universidad Miguel Hernández de Elche.

Lineas de investigación

Historia y Filosofía de la ciencia, epistemología de la geometría espacio-temporal y la causalidad cuántica

\section{Publicaciones recientes}

Alemañ, R. 2008: Quantum Mechanics versus special relativity: a forgotten conflict. En Phil-Sci Archive (Center for Philosophy of Science - Pittsburgh University) http://philsci-archive. pitt.edu/archive/00004313/. Tambien en Philpapers beta - Online Research in Philosophy.

Alemañ, R. 2008: “Geometría y Física: De Hertz a Einstein”, Llull - Revista de la Sociedad Española de Historia de las Ciencias y la Tecnología (SEHCyT), vol. 31, nº 68 (2008), pp. 189-207.

\section{Dirección postal}

Avda. Universidad s/n. Edif. Torrevaillo, 03202-Elche

\section{Dirección electrónica}

raalbe.autor@gmail.com 